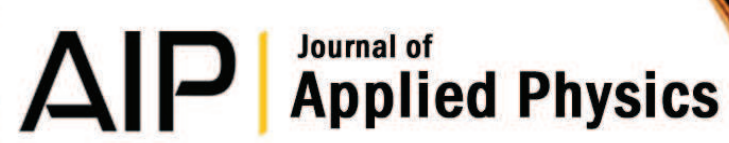

\section{Stability conditions, nonlinear dynamics, and thermal runaway in microbolometers}

G. B. Brandão, L. A. L. de Almeida, G. S. Deep, A. M. N. Lima, and H. Neff

Citation: J. Appl. Phys. 90, 1999 (2001); doi: 10.1063/1.1384852

View online: http://dx.doi.org/10.1063/1.1384852

View Table of Contents: http://jap.aip.org/resource/1/JAPIAU/v90/i4

Published by the American Institute of Physics.

\section{Related Articles}

Description of transport mechanisms in a very long wave infrared quantum cascade detector under strong magnetic field

J. Appl. Phys. 112, 123705 (2012)

Combined cross sectional scanning tunneling microscopy and high resolution x-ray diffraction study for quantitative structural descriptions of type-II superlattice infrared detectors Appl. Phys. Lett. 101, 241908 (2012)

A low noise, nonmagnetic fluorescence detector for precision measurements Rev. Sci. Instrum. 83, 113105 (2012)

Polarity inversion and coupling of laser beam induced current in As-doped long-wavelength HgCdTe infrared detector pixel arrays: Experiment and simulation

Appl. Phys. Lett. 101, 181108 (2012)

Infrared detectors based on semiconductor $\mathrm{p}-\mathrm{n}$ junction of $\mathrm{PbSe}$

J. Appl. Phys. 112, 086101 (2012)

\section{Additional information on J. Appl. Phys.}

Journal Homepage: http://jap.aip.org/

Journal Information: http://jap.aip.org/about/about_the_journal

Top downloads: http://jap.aip.org/features/most_downloaded

Information for Authors: http://jap.aip.org/authors

\section{ADVERTISEMENT}

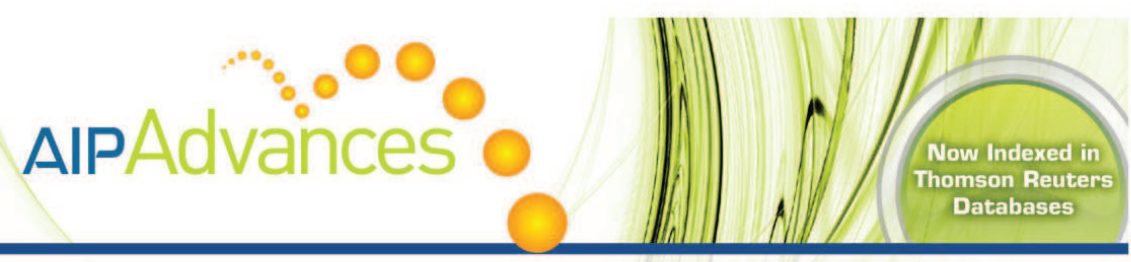

\section{Explore AIP's open access journal: Rapid publication \\ - Article-level metrics \\ Post-publication rating and commenting}




\title{
Stability conditions, nonlinear dynamics, and thermal runaway in microbolometers
}

\author{
G. B. Brandão ${ }^{a)}$ \\ Department of Electrical Engineering, Universidade Federal da Paraíba, 58109-970, Campina Grande, \\ PB, Brazil
}

L. A. L. de Almeida ${ }^{\text {) }}$

Department of Electrical Engineering, Universidade Federal de Paraíba, 58109-970, Campina Grande, PB, Brazil and Department of Electrical Engineering, Universidade Federal da Bahia, Bahia, Brazil

G. S. Deep and A. M. N. Lima

Department of Electrical Engineering, Universidade Federal de Paraíba, 58109-970, Campina Grande, PB, Brazil

H. Neff ${ }^{\mathrm{C}}$

Department of Electrical Engineering, Universidade Federal da Paraíba, 58109-970, Campina Grande, PB, Brazil and VIR-TECH A/S, Kuldyssen 10, DK-2630 Taastrup, Denmark

(Received 4 December 2000; accepted for publication 17 May 2001)

\begin{abstract}
The nonlinear dynamic behavior of microbolometers, operating at room temperature $(300 \mathrm{~K})$ under conditions of positive electrothermal feedback is investigated. An improved device model, based on the heat balance equation is developed. It takes into account the temperature dependence of the thermophysical parameters, such as thermal coupling coefficient between the sensor and its surroundings, and sensor heat capacity and its thermal resistance coefficient. Operational considerations for thermoresistive microbolometer with positive and negative temperature coefficient of resistance are discussed for both, constant current and constant voltage modes of operation. Analytical expressions are derived for predicting stable and unstable operation. Safety factors $L_{0}$, establishing the biasing conditions for stable device operation are proposed for the positive temperature coefficient of resistance and negative temperature coefficient of resistance type sensors. Limits for fast catastrophic destruction are provided, and the dynamic characteristics of the associated thermal runaway phenomenon is illustrated. This effect, as predicted by analysis and numerical simulation, was observed experimentally, confirming the validity of the proposed modeling approach for the microbolometer. (C) 2001 American Institute of Physics.
\end{abstract}

[DOI: $10.1063 / 1.1384852$ ]

\section{INTRODUCTION}

Recent technological progress in microtechnology has resulted in a significant improvement of microsensor sensitivity. Microstructured thermal radiation sensors, with operation extending into the far infrared region, have attracted considerable technological and economic interest.

Commonly employed far infrared (FIR) photon detectors, based on the cadmium-mercury-telluride (CMT) compound semiconductor system, typically reveal a sharp cutoff wavelength at around $12-14 \mu \mathrm{m}$, depending on composition, beyond which sensor performance rapidly deteriorates. In contrast, thermal or bolometric sensors are spectrally broadband and their optical sensitivity range may extend to wavelengths of nearly $100 \mu \mathrm{m}$. It is solely defined by the properties of the optical absorber, employed in the design of thermal microsensors.

The performance of cooled microbolometers, operating slightly above the boiling point of liquid nitrogen, now

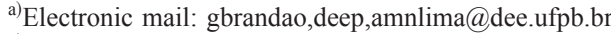

${ }^{b)}$ Electronic mail: lalberto@ufba.br

${ }^{c}$ Electronic mail: hn@vir-tech.dk
}

nearly matches that of quantum detectors, ${ }^{1,2}$ particularly at wavelengths $>10 \mu \mathrm{m}$. Uncooled microbolometers, operating at room temperature, as are investigated in this work, typically reveal a reduced performance in the FIR wavelength region, as compared to devices operating at low temperatures. However, the achievable sensitivity $D^{*}$ of state of the art uncooled microbolometers can now be considered to be superior to that of FIR-CMT quantum detectors, when operated at room temperature.

Due to the specific design and structural features of microbolometers, special care is required to establish reliable operation and excessive thermal load should be avoided. So far, there does not exist a full physical understanding, regarding the effects and limitations of thermal loads, imposed by electrical bias of the microbolometer.

Optimization of the thermal budget of the device, in connection with the given electrical bias and operating conditions, thus represents a critical issue. There basically exist four different operating modes for microbolometers. The first mode involves biasing the device with a fixed current through the sensor, with voltage drop across the sensor as an output signal, which is denoted as the constant current mode 
TABLE I. Biasing modes and its effects on operating characteristics of microbolometers.

\begin{tabular}{lcccc}
\hline \hline Sensor & Biasing & Electrothermal feedback & Thermal response time & Operational feature \\
\hline PTC & CVM & Negative & Decreases & Stable \\
PTC & CCM & Positive & Increases & Potentially unstable \\
NTC & CVM & Positive & Increases & Potentially unstable \\
NTC & CCM & Negative & Decreases & Stable \\
\hline \hline
\end{tabular}

(CCM). The second mode consists in biasing the device with a fixed voltage, with the current as an output signal, that is denoted as the constant voltage mode (CVM). Finally, exists active modes exist where either power or temperature of the sensor is kept constant, using feedback control principles and these modes are referred to as CPM and CTM, respectively. The CPM is not yet used in practical applications whereas CTM has been widely employed.

All these possible operating modes affect bolometric device performance differently. Among other quantities, it involves: the dynamic and frequency ranges, the extent of linear operating region, and sensitivity. Despite some serious drawbacks, historically the CCM operation of bolometric sensors is the common mode of operation and widely employed. Recently, the CVM has attracted technical interest for the cooled transition edge microbolometers. ${ }^{2-4}$ Both, simulation and experimental results have indicated the advantages of constant voltage biasing in terms of frequency response and linear operating range, while only limited changes were found for sensitivity. A further interesting aspect is its compatibility with charge coupled device read out.

The association of a particular biasing mode and the physical properties of the heat sensing element may yield nonlinear microbolometer operating characteristics. It is due to the influence of Joule self-heating and the possible temperature dependence of critical thermophysical quantities as: the temperature coefficient of resistance (TCR), thermal coupling coefficient of the sensor with its surroundings $(G)$, and the heat capacity $(C)$ of the device arrangement. Thus, nonlinear effects are pronounced for transition edge devices, where the TCR varies considerably with temperature. The prominent figures of merit as thermal response time, responsivity, linearity range, and detectivity are crucially affected by the biasing conditions, ${ }^{2,5}$ employed for the sensor.

In this work, thermoresistive sensor nonlinearities are attributed primarily to Joule self-heating, which causes electrothermal feedback in the sensor operation. The biasing induced change of the resistance of the heat sensing element interferes with the radiation induced resistance variation. As an example, in the presence of incident radiation, for the CVM the dissipation of Joule heat in the thermosensitive element of the microbolometer is $J_{\mathrm{CVM}} \mp \Delta J_{\mathrm{CVM}}=V_{\text {bias }}^{2} /\left[R_{0}\right.$ $\left.\pm \Delta R\left(\beta, T_{s}\right)\right]$ while for $\mathrm{CCM}$ is $J_{\mathrm{CCM}} \pm \Delta J_{\mathrm{CCM}}=I_{\text {bias }}^{2}\left[R_{0}\right.$ $\left.\pm \Delta R\left(\beta, T_{s}\right)\right]$. It is worth observing that the signs of $\Delta J$ terms in the two expressions depend on the sign of the thermal coefficient of resistance $(\beta)$ of the heat sensing material. In case of positive feedback, $\Delta J$ and the external radiation power add up. In case of negative feedback, $\Delta J$ subtracts from incident radiation power and may even cancel each other. This would keep the sensor temperature at the original temperature, even in presence of external radiation. This is attributed to the existence of strong negative electrothermal feedback. Microbolometer performance is very much affected, and considerably differ from one mode of operation to the other.

Mathematically, the dynamic characteristics of thermoresistive sensors are adequately described by the heat balance equation. The traditionally used, first order linear differential equation, neglecting Joule heating, ${ }^{6}$ yields a simple exponential solution. However, under certain bias conditions coupled with the use of temperature dependent thermophysical sensor parameters, makes this equation a nonlinear one. Among other features, its solution reveals a singularity.

An important result, implying a pronounced effect on device operation, is the possibility of the loss of stability. Experimentally, it was observed that under certain biasing conditions, the sensor temperature begins to continuously increase, even in the absence of external radiation or heating. In practice, for some electrical bias, instead of reaching a final steady state the sensor temperature keeps varying at an almost constant rate of the order of some $\mathrm{mC} / \mathrm{s}$. This is known as thermal drift and under this condition the output data is erroneous.

Eventually, with further increase in the electrical bias, uncontrolled thermal runaway will result in explosive heat up, leading to catastrophic destruction of the device. Hence, commercially available thermoresistive microbolometers need to be equipped with a protective circuit at the bias input terminals, when operated under conditions of positive electrothermal feedback, to avoid destructive electrical biasing conditions.

The direct equivalent electrical $R-C$ analog representation of the microbolometer, fails to describe the aforementioned features of thermoresistive sensors. ${ }^{6}$ It should be noted that there are no clear rules available in the open literature that could provide practical usable technical guidelines, regarding the limits of electrical bias of thermoresistive sensors to hold for both, stable operation and to avoid possible catastrophic sensor failure. Table I illustrates the relation between biasing modes and operational characteristics of microbolometers. This table illustrates the conditions for existence of positive electrothermal feedback. It is worth noting that a PTC (positive temperature coefficient of resistance) sensor operating in the CCM as well as a NTC (negative temperature coefficient of resistance) sensor operating in the CVM involve positive electrothermal feedback.

In this work, an attempt is made to identify the physical origin of the aforementioned operational characteristics of microblometers. The specific conditions and the biasing limits, required for stable sensor operation, considering tempera- 
ture dependent thermophysical parameters, have been evaluated in detail. The occurrence, necessary conditions for and dynamics of the thermal runaway phenomenon in electrically biased thermoresistive sensors have been evaluated by simulation and experiments. From these data, the limiting bias margins have been defined, beyond which sensor operation is severely affected.

The article is organized as follows: we first illustrate the origin of thermoresistive sensor instability from a mathematical point of view. This is observable from the closed form solution of the heat balance equation, describing the physical operation of an electrically biased thermoresistive sensor. Based on these findings, a refined sensor model is developed, taking into account the specific characteristics of the relevant thermophysical sensor parameters.

Generally, all thermophysical sensor parameters vary with sensor temperature and surrounding conditions of the device under consideration. Commonly, for mathematical simplification, such effects are neglected.

Finally, reliable safety margins are proposed for two types of thermoresistive sensors, operating under conditions of positive electrothermal feedback, where singular conditions can occur.

\section{THEORETICAL ASPECTS AND MODEL}

As mentioned before, the origin of thermal instability is due to positive electrothermal feedback, resulting from electrical sensor biasing. Under the conditions of negative electrothermal feedback, the effective thermal sensor response time is reduced and its operation is always stable within the maximum ratings. ${ }^{2}$ For positive electrothermal feedback, in contrast, response time and sensor temperature may increase to values that exceed the range of practical use.

Two different approaches have been used for simulation. First, the thermal coupling coefficient $G$ and thermal capacity $C$ are treated as fixed values, independent of the sensor temperature. This is a common approach for formal treatment of thermoresistive sensors. ${ }^{6}$ Second, in a more realistic approach, the dependence of $G$ and $C$ on the actual sensor temperature is included.

The limitations, resulting from thermal sensor instability, can be analyzed with the help of the heat balance equation. ${ }^{7}$ For a thermoresistive sensor, biased with electrical power $J_{\mathrm{CVM}}=V_{b}^{2} / R_{s}$ or $J_{\mathrm{CCM}}=I_{b}^{2} R_{s}$, the thermodynamic equilibrium of the sensor can be described by

$$
C \frac{d\left(T_{s}-T_{a}\right)}{d t}=\left(J_{\mathrm{CVM}} \text { or } J_{\mathrm{CCM}}\right)-G\left(T_{s}-T_{a}\right)+\epsilon P,
$$

where $\epsilon P$ is the absorbed radiation power, $I_{b}$ and $V_{b}$ are bias current and voltage, respectively and $T_{a}$ is the heatsink temperature assumed to remain constant.

\section{A. Mathematical analysis and sensor dynamics}

As mentioned before, there are four different operating modes for microbolometers. In this work, only the effects related with the CCM and the CVM will be investigated.

\section{Constant current mode}

The basic dynamic characteristics of an electrically biased thermoresistive sensor can be illustrated by analyzing the solution of the associated linear heat balance equation, as is briefly outlined later.

The relationship between sensor temperature $T_{s}$ and its resistance, $R_{s}$, can be approximated by $R_{S} \approx R_{0}\left(1+\beta T_{s}\right)$, where $R_{0}$ is the sensor resistance at $0 \mathrm{C}$. This expression is accurate for the PTC sensor and reasonably good, over a limited range of temperature, for the NTC sensor. For simplification all, $G, C$, and heatsink temperature $T_{a}$ are assumed to be constant. This approach allows an analytical solution of Eq. (1) for CCM operation. The evolution of the $T_{s}(t)$ for a step change in the incident radiation, i.e., $\epsilon P(t)$ $=\epsilon P_{0}, \forall t>0$, on a already biased sensor is described by

$$
T_{s}(t)=\frac{I_{b}^{2} R_{0}+G T_{a}+\epsilon P_{0}\left(1-e^{-t / \tau_{\mathrm{CCM}}}\right)}{G-I_{b}^{2} R_{0} \beta},
$$

where $\tau_{\mathrm{CCM}}=C /\left(G-I_{b}^{2} R_{0} \beta\right)$.

In the earlier equation, when $I_{b}^{2}=G /\left(\beta R_{0}\right)$ for positive $\beta$ (PTC), the sensor temperature $T_{s}(t) \rightarrow \infty$ and $\tau_{\mathrm{CCM}} \rightarrow \infty$ and at this bias current, thermal runaway would occur as a result of strong positive electrothermal feedback. For negative $\beta$ (NTC), there is negative electrothermal feedback and the denominator always remains positive and consequently uncontrolled thermal runaway and catastrophic sensor failure would not occur. In fact the sensor operation is unconditionally stable.

\section{Constant voltage mode}

For CVM operation, Eq. (1) becomes nonlinear and there is no closed form solution available. To overcome this difficulty we make the following approximation:

$$
J_{\mathrm{CVM}}=\frac{V_{b}^{2}}{R_{0}\left(1+\beta T_{s}\right)} \approx\left(\frac{1}{R_{0}}-\frac{\beta T_{s}}{R_{0}}\right) V_{b}^{2}
$$

and Eq. (1) becomes

$$
C \frac{d T_{s}}{d t}=\left(\frac{1}{R_{0}}-\frac{\beta T_{s}}{R_{0}}\right) V_{b}^{2}-G\left(T_{s}-T_{a}\right)+\epsilon P .
$$

In this format, an analytical solution is available. For constant $G, C$, and heatsink temperature $T_{a}$, the evolution of the $T_{s}(t)$ for a step change, $\epsilon P_{0}$, in the incident radiation on a already biased sensor is given by

$$
T_{s}(t)=\frac{V_{b}^{2}+G T_{a} R_{0}+\epsilon P_{0} R_{0}\left(1-e^{-t / \tau_{\mathrm{CVM}}}\right)}{V_{b}^{2} \beta+G R_{0}},
$$

where $\tau_{\mathrm{CVM}}=C R_{0}\left(V_{b}^{2} \beta+G R_{0}\right)$.

For NTC sensors $\beta$ is negative and for a certain value of $V_{b}$ the denominator can vanish, making $T_{s}(t) \rightarrow \infty$, and time constant $\tau_{\mathrm{CVM}}$ also becomes infinity. Actually, with an NTC sensor operating in the CVM, there exists positive electrothermal feedback and for $V_{b}^{2}=-G R_{0} / \beta$ destructive thermal runaway is imminent. For positive $\beta$ (PTC), the denominator always remains positive, and the thermal runaway phenom- 
enon does not occur because there exists negative electrothermal feedback and the sensor operation is unconditionally stable.

\section{B. Steady state}

Stable steady-state operation in both modes is achieved if the electrical bias is kept below the critical values, i.e., $I_{b}^{2}=G /\left(\beta R_{0}\right)$ and $V_{b}^{2}=-G R_{0} / \beta$, respectively. However, catastrophic failure occurs for a bias smaller than but close to these critical values, if the sensor temperature exceeds the melting point of the sensor material.

Furthermore, the steady-state sensor temperature sensitivity as derived from Eq. (2) can be verified to depend on the bias current (CCM). It increases with bias current for the PTC sensor and decreases for the NTC sensor. This is directly related to sign of electrothermal feedback existing in the sensor operation.

For the CVM, the earlier sensitivity, as derived from Eq. (4) also depends upon the bias voltage. It increases with bias voltage for the NTC sensor and decreases for the PTC sensor. Again, this is directly related to sign of electrothermal feedback existing in the sensor operation.

\section{Response time}

The thermal time constant $\tau_{\mathrm{CCM}}$ and $\tau_{\mathrm{CVM}}$ differ from the intrinsic thermal time constant as defined by $\tau_{\text {intr }}$ $=C / G$. It is easy to observe that both $\tau_{\mathrm{CCM}}$ and $\tau_{\mathrm{CVM}}$ increase for positive electrothermal feedback and decrease in the case of negative electrothermal feedback, due to electrical bias.

\section{Sensor drift}

It should be noted, however, that the mathematical approach, as outlined earlier, does not explain the experimentally observed slow drift of the output signal or lack of stable steady-state conditions, under certain bias conditions. This consistently observed second order phenomenon, was attributed tentatively to a slow increase of the heat sink temperature $T_{a}$. It results from both, the limited heat capacity of any real heatsink in a sensor arrangement and lack of control to maintain $T_{a}$ constant. Thermoresistive bolometers, operating at room temperature, commonly are not equipped with an external temperature control, primarily for economic reasons. Hence, under physically realistic conditions, $T_{a}$ in the heat balance Eq. (1) cannot be taken as a fixed or well defined quantity. However, the variation of $T_{a}$ cannot be accounted for directly. To mathematically treat this problem in an equivalent form, $T_{a}$ can be considered to remain constant and $G$ and $C$ are assumed to vary with sensor temperature $T_{s}$. The heat transfer from the sensor element, and steady warming up of the surrounding heatsink has been verified recently by finite element calculations for a superconducting membrane based transition edge microsensor. ${ }^{8}$

When $G$ and $C$ are considered to be varying with sensor temperature, the determination of $T_{s}, I_{b}^{2}$, and $V_{b}^{2}$ at the singularity condition is more difficult. The solution of the heat balance equation would require an iterative procedure. To overcome this problem, Eq. (1), was solved numerically for

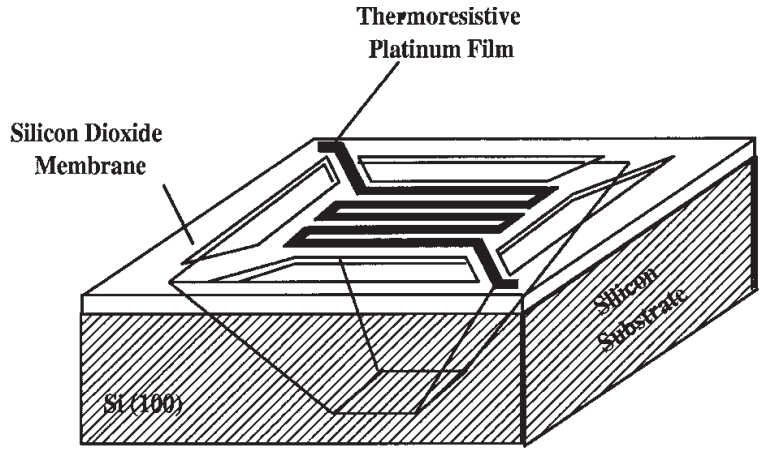

(a)

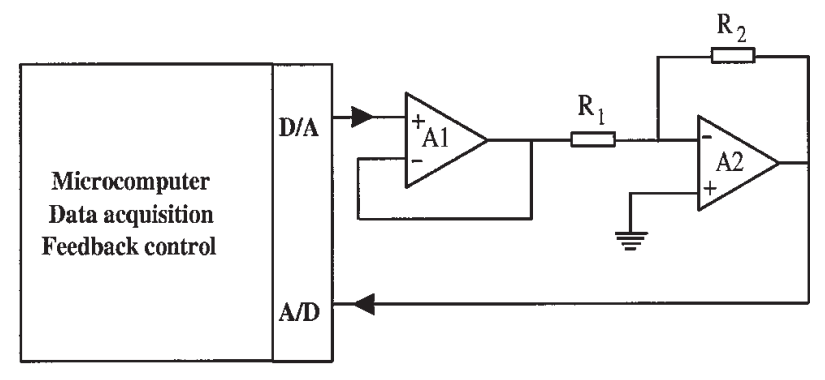

(b)

FIG. 1. Microbolometer structure and block diagram of the electronic circuit used in experimental tests.

two different cases. For case 1, temperature independent thermophysical parameters were assumed. For case 2, experimentally determined temperature independent thermophysical parameters were employed.

The operational characteristics of the NTC and the PTC sensors operating in the CCM and the CVM, as outlined before, are summarized in Table I.

\section{THERMOPHYSICAL PARAMETERS}

In the study of thermal stability of microbolometers, $G$ and $C$ are usually considered constant. In this investigation an attempt is made to establish a methodology for determining the temperature dependence of these parameters for different sensor configurations.

Two different sensors have been investigated. First, the micromachined sensor, shown in Fig. 1(a) that employs a thin platinum film, deposited onto a $\mathrm{SiO}_{2}$ membrane. It is designed to operate at room temperature, exhibits a positive TCR of $0.00348 \mathrm{C}^{-1}$ and has a nominal resistance of $15 \mathrm{k} \Omega$. The membrane has been suspended from the surrounding silicon frame with four legs, located across the four corner points. Its intrinsic thermal time constant $\tau_{\text {intr }} \approx 1 \mathrm{~ms}$ at ambient temperature, in still air. Details on the design and fabrication of the microbolometer are given in Ref. 9. The second sensor under investigation is a thermistor, fabricated from a semiconducting metaloxide. Its nominal resistance is approximately $5 \mathrm{k} \Omega$, the intrinsic time constant $\tau_{\text {intr }} \approx 3.4 \mathrm{~s}$, in still air, and the negative TCR is $0.0448 \mathrm{C}^{-1}$ at room temperature. 

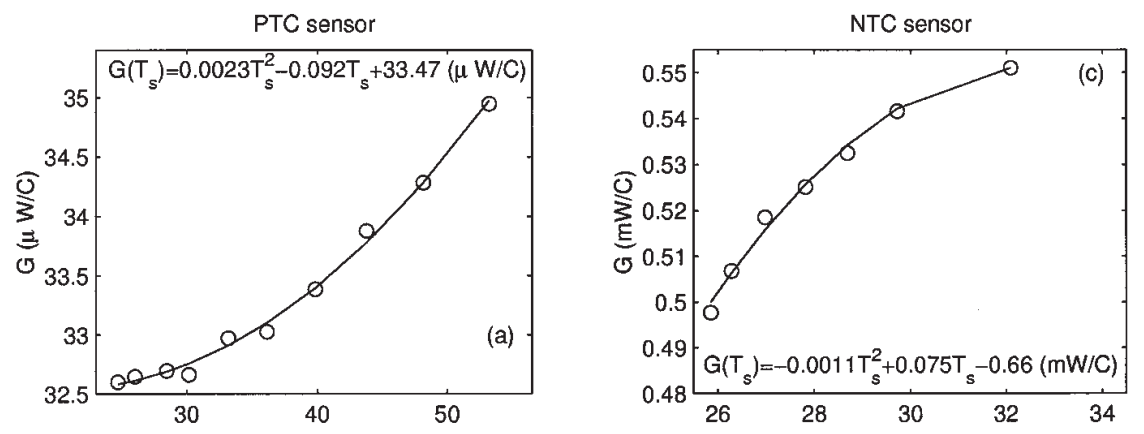

FIG. 2. Experimentally determined thermal coupling coefficient of the sensor with its surroundings $G\left(T_{\mathrm{s}}\right)$ and heat capacity $C\left(T_{s}\right)$ of the PTC
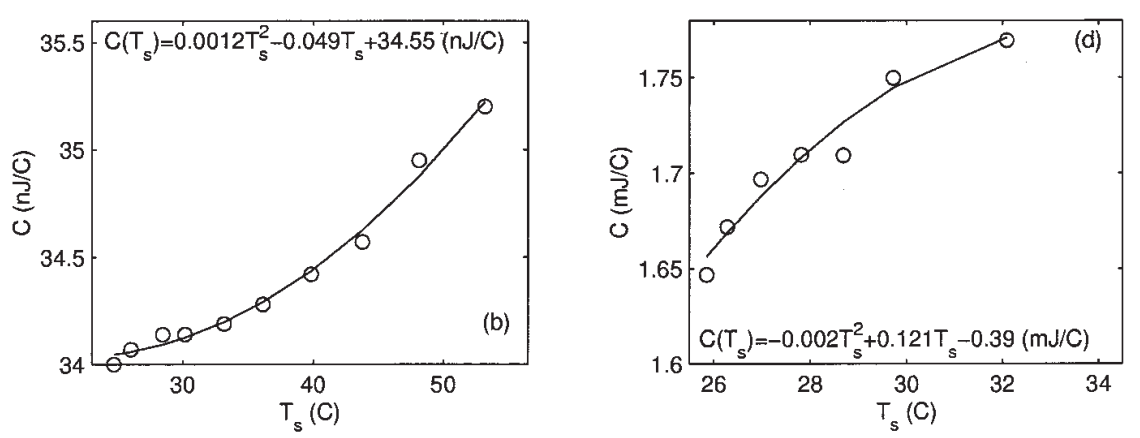
sensor $[2(a), 2(b)]$ and the NTC sensor [2(c), 2(d)].

Apart from the limited heat capacity of the surrounding heatsink, accounting for a slow change of its temperature $T_{a}$ with time, in response to the variation of $T_{s}$, there also exists an intrinsic temperature dependence of the thermophysical sensor parameters. Both, the thermal conductivity $\kappa$ and specific heat $c_{p}$ of the sensor material also vary with temperature. Most polycrystalline metals reveal a very small decreases of $\kappa$ with temperature at room and higher temperatures exhibiting a maximum at $T_{s} \sim 50 \mathrm{~K}$. On the other hand, $c_{p}$ increases as $T_{s}^{3}$ at very low $T_{s}$, and increases approximately linearly at temperatures above the Debye temperature. For details, concerning the thermophysical properties of solids, the reader may refer to Ref. 10 .

The variations of $G$ and $C$ with sensor temperature $T_{s}$ were determined experimentally operating the sensor in the constant power mode (CPM), i.e., $J_{\mathrm{CVM}}(t)=J_{\mathrm{CCM}}(t)$ $=J_{0}, \forall t$, for different levels of Joule power. This was implemented using feedback control, to eliminate the effect of electrothermal feedback. ${ }^{11}$ Under this condition, Eq. (1) becomes $G\left(T_{s}\right)=\left(J_{\mathrm{CVM}}\right.$ or $\left.J_{\mathrm{CCM}}\right) /\left(T_{s}-T_{a}\right)$, from which values of $G$ for different values of $T_{s}$ are obtained. Eventually, $C\left(T_{s}\right)$ can be determined, using $C\left(T_{s}\right)=\tau_{\text {intr }} G\left(T_{s}\right)$ and the experimentally obtained intrinsic thermal time constant, by maintaining dynamically the Joule power in the sensor constant. The sensor is initially excited by a current step. ${ }^{12}$

The experimentally determined temperature dependence of $G\left(T_{s}\right)$ and $C\left(T_{s}\right)$ of the PTC sensor are

$$
G\left(T_{s}\right)=\left(0.0023 T_{s}^{2}-0.092 T_{s}+33.47\right) 10^{-6}(\mathrm{~W} / \mathrm{C}),
$$

and

$$
C\left(T_{s}\right)=\left(0.0012 T_{s}^{2}-0.049 T_{s}+34.55\right) 10^{-9}(\mathrm{~J} / \mathrm{C}) .
$$

As experimentally verified, the TCR is independent of $T_{s}$ up to temperatures of $500 \mathrm{C}$ for the PTC sensor.

The experimentally determined temperature dependence of $G\left(T_{s}\right)$ and $C\left(T_{s}\right)$ for the NTC device are

$$
G\left(T_{s}\right)=\left(-0.0011 T_{s}^{2}+0.075 T_{s}-0.66\right) 10^{-3}(\mathrm{~W} / \mathrm{C})
$$

and

$$
C\left(T_{s}\right)=\left(-0.002 T_{s}^{2}+0.121 T_{s}-0.39\right) 10^{-3}(\mathrm{~J} / \mathrm{C}) .
$$

The experimentally determined temperature dependence of the thermal resistance coefficient of the NTC sensor

$$
\beta=\frac{1}{R_{s}} \frac{d R_{s}}{d T_{s}}=-\frac{3774}{T_{s}^{2}} .
$$

The schematic of the electrical circuit used in the experimental tests is outlined in Fig. 1(b). The electrical circuit is configured in the following way: CCM: $R_{1}=R_{\mathrm{FIXED}}$ and $R_{2}$ $=R_{s}$, CVM: $R_{1}=R_{s}$ and $R_{2}=R_{\mathrm{FIXED}}$ and CPM: $R_{1}$ $=R_{\text {FIXED }}$ and $R_{2}=R_{s}$. Note that the resistance of $R_{\text {FIXED }}$ does not vary with temperature, and $R_{s}$ represents the thermoresistive device. All the experiments were conducted with a microcomputer based platform, employing a multifunction 12 bit data acquisition card from Advantest Incorporated.

Experimentally determined variations of $G$ and $C$ with sensor temperature $T_{s}$ for a PTC and NTC sensor are displayed in Figs. 2(a)-2(d). Both $G$ and $C$ increase monotonically with sensor temperature for the PTC sensor as shown in Figs. 2(a)-2(b). These findings agree with the results of earlier work on the same device, where a different experimental approach was used for thermal parameter extraction. ${ }^{13}$ For 

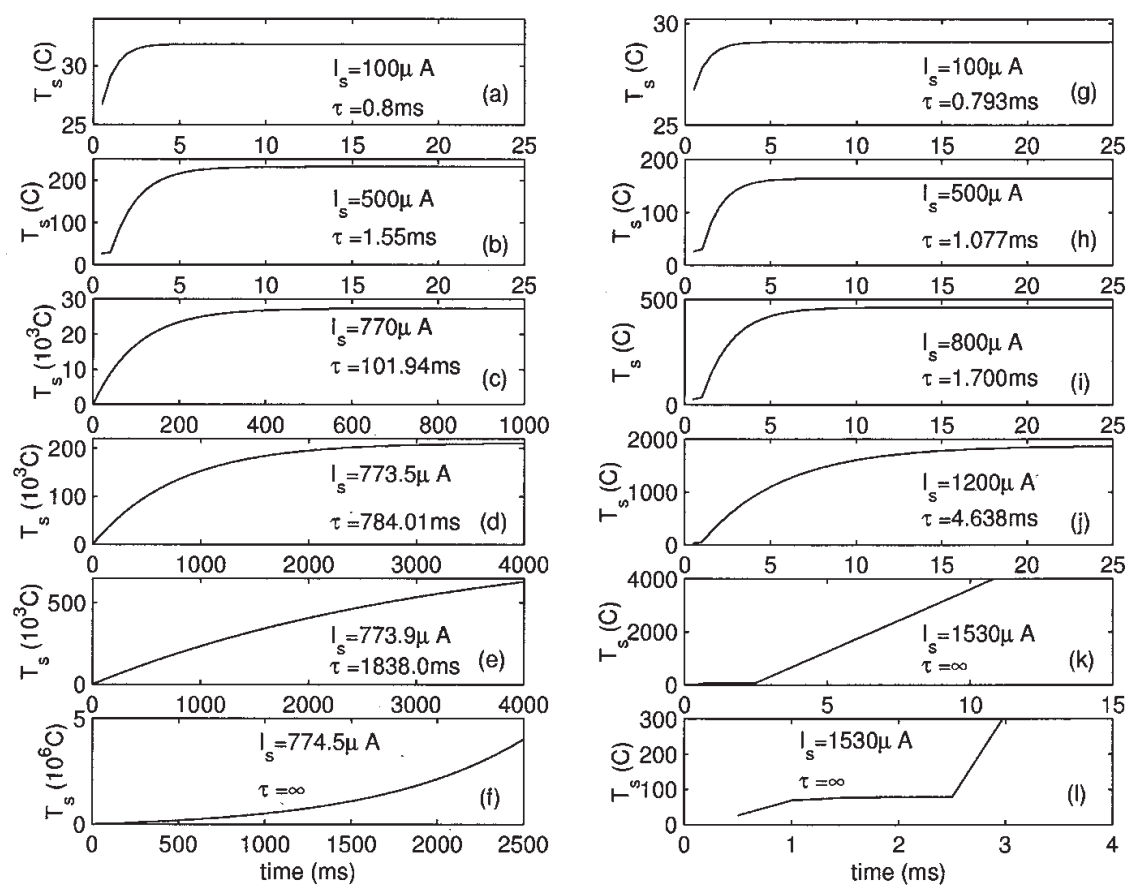

FIG. 3. Evolution of sensor temperature $T_{s}$ for the PTC sensor at different bias currents and $100 \mu \mathrm{W}$ step incident radiation (simulation). Figures 3(a)-3(f) refer to case 1, with constant $G$ and $C$. Figures $3(\mathrm{~g})-3(\mathrm{l})$ are for case 2, with temperature dependent $G\left(T_{s}\right)$ and $C\left(T_{s}\right)$ [as depicted in Figs. 2(a) $-2(\mathrm{~d})]$. The effective time constant for each bias current is indicated in the figures.

the NTC sensor, the measured $G$ and $C$ are shown in Figs. 2(c)-2(d), and similar temperature dependence of $G$ and $C$ was observed.

\section{STABILITY CRITERION}

Based on the aforementioned considerations, concerning the singularities of Eqs. (2) and (4), margins and limits of the maximum applicable bias current and voltage most conveniently can be defined, using the safety factor $L_{0}$, together with the singularity conditions of Eqs. (2) and (4). For CCM operation

$$
I_{\max }=L_{0} \sqrt{G / \beta R_{0}}
$$

and for CVM operation

$$
V_{\max }=L_{0} \sqrt{-G R_{0} / \beta},
$$

with the safety factor $0<L_{0}<1$.

As mentioned before, we are not aware of the existence of a clear rule, regarding the appropriate choice of $L_{0}$ in the literature, although, values of 0.3 have been reported for high $T_{c}$ transition edge devices ${ }^{1,2,14}$ and 0.5 for a microbolometer fabricated using porous silicon membranes. ${ }^{15}$ These devices with positive TCR were operated in the CCM mode, thus implying positive electrothermal feedback.

\section{RESULTS AND DISCUSSION}

\section{A. Constant current operation}

Figures 3(a)-3(f) illustrate the numerically determined evolution of $T_{s}(t)$ [Eq. (1)] for the PTC sensor. CCM biasing causes positive electrothermal feedback, for a step change of $100 \mu \mathrm{W}$ in the incident radiation. In these plots, constant values of $G$ and $C$ have been employed (case 1). The constant $G$ and $C$ values are $3.25 \times 10^{-5} \mathrm{~W} / \mathrm{C}$ and 3.4 $\times 10^{-8} \mathrm{~J} / \mathrm{C}$, respectively, for the PTC sensor. For the NTC device, the constant $G$ and $C$ values are $0.525 \times 10^{-3} \mathrm{~W} / \mathrm{C}$ and $1.6 \times 10^{-3} \mathrm{~J} / \mathrm{C}$, respectively.

Figures $3(\mathrm{~g})-3(\mathrm{l})$ present the corresponding simulation results, for temperature dependent $G\left(T_{S}\right)$ and $C\left(T_{S}\right)$ (case 2 ). For moderate bias current, $T_{s}(t)$ varies exponentially with time. Above the critical bias current in both the cases, catastrophic thermal runaway is observable. For constant $G$ and $C$, the runaway phenomenon appears smoothly with an approximately exponential increase for bias current $>774.5$ $\mu \mathrm{A}$, about $1 \mathrm{~s}$ after application of the radiation step. The result of numerical solution of Eq. (1), agrees within 1\% with the value calculated using the formula, based on the singularity of $T_{s}(t)$. Assuming constant $G$ and $C$, the calculated value of the bias current causing runaway would be $I_{\max }=783 \mu \mathrm{A}$ for $R_{0}=13840 \Omega$.

For the conditions of case 2, as expected, thermal runaway sets in at a significantly higher bias current of $1530 \mu \mathrm{A}$ [see Fig. 3(k)]. For both the cases, the thermal time constant continuously increases with bias current and approaches $\infty$ under thermal runaway conditions. The sensor film temperature is much higher for case 1 , as compared to that for case 2 for the same bias conditions, and exceeds the melting point of the platinum film at a bias current $>500 \mu \mathrm{A}$, as illustrated in Fig. 3(c). This was not verified experimentally. The thermal run away for case 2, as displayed in Fig. 3(k), occurs at a bias current of $1530 \mu \mathrm{A}$. The details of this figure, for the time interval of 1-4 $\mathrm{ms}$ is shown in Fig. 3(1). The thermal runaway sets in about $2.5 \mathrm{~ms}$ after the application of radiation excitation at $t=0$. The rate of temperature evolution in this case is greater than that in case 1 . The melting point of platinum would be reached after about $7 \mathrm{~ms}$. A higher bias current of the order of $1200 \mu \mathrm{A}$ as compared to case 1 , can be employed, without detrimental damage to the sensor as 


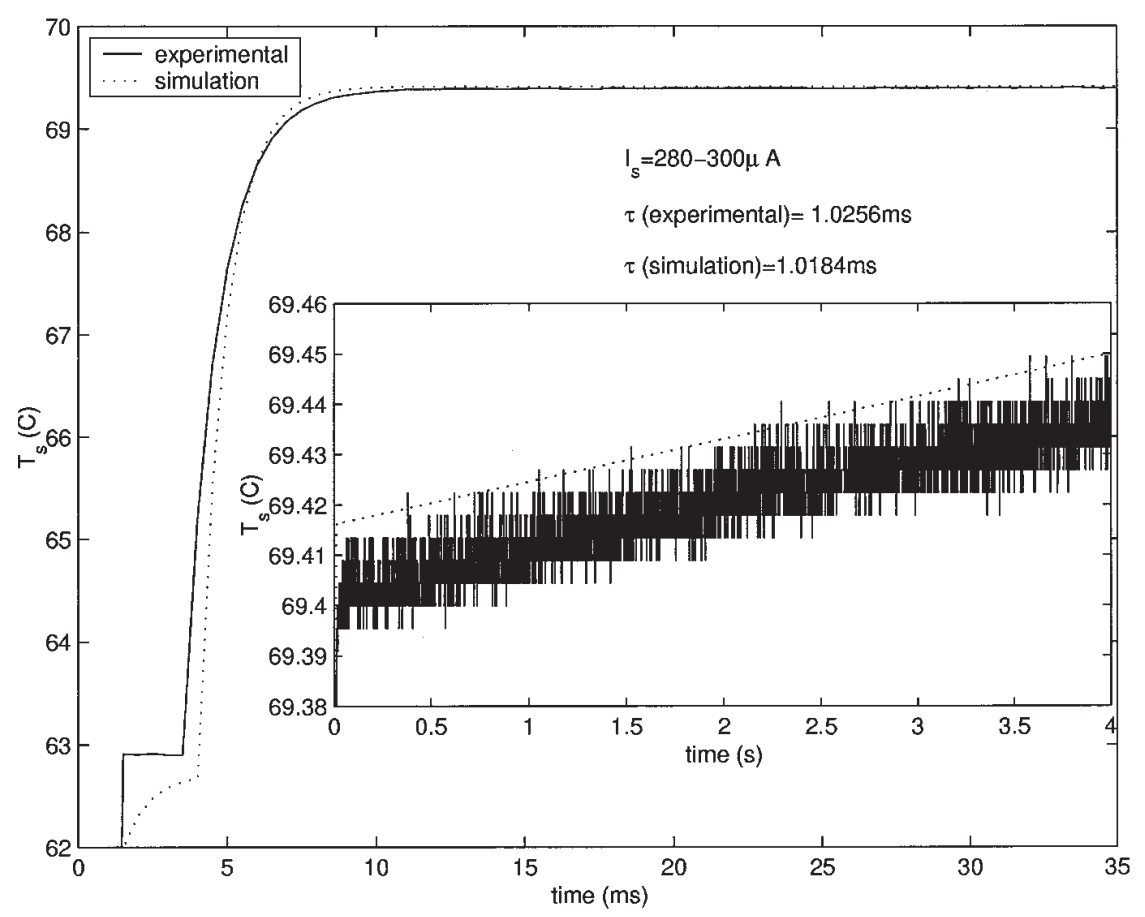

FIG. 4. Experimental (solid line) and calculated response curve (broken line) of the PTC sensor, with step excitation of $20 \mu \mathrm{A}$ on the top of 280 $\mu \mathrm{A}$. Inset illustrates the appearance of output signal drift of $8 \mathrm{mC} / \mathrm{s}$ under this condition.

shown in Fig. 3(j). The thermal time constant increases about a factor of 6 to $\tau=4.638 \mathrm{~ms}$.

In practice, the maximum usable bias current, to maintain stable device operation, should be set at a much lower value. In Fig. 4 the temporal evolution of the sensor temperature in response to a current step in absence of radiation, obtained both experimentally as well as by numerical solution of Eq. (1), are shown. The simulated and experimental responses are in agreement within $1 \% . T_{s}(t)$ increases exponentially with a thermal time constant $\tau=1.025 \mathrm{~ms}$ in experimental response, and $1.018 \mathrm{~ms}$ in the simulated response. The inset of Fig. 4 illustrates the details of the curve around $69 \mathrm{C}$. For $I_{b}>280 \mu \mathrm{A}$ a steady linear increase, or drift of the platinum film temperature can be observed. $T_{s}(t)$ increases approximately linearly at a rate of about $8 \mathrm{mC} / \mathrm{s}$. Experimentally, it has been observed that the rate of drift increases with bias current.

\section{Sensor stability conditions}

The appearance of temperature drift in the output signal can be used, to define the limiting condition for the maximum permitted bias current. Since the thermophysical quantities $G\left(T_{S}\right)$ and $C\left(T_{S}\right)$ commonly are not available without performing extensive experimental sensor characterization, the operational limits are determined from Eq. (10) and (11) for constant $G$ and $C$. In case of the PTC sensor operating in CCM mode, $L_{0}=280 \mu \mathrm{A} / I_{\max }=0.35$, the upper limit of bias current $I_{\max }^{\text {stable }}$ may be defined as

$$
I_{\max }^{\text {stable }} \approx 0.35 \sqrt{G / \beta R_{0}}
$$

for which a stable sensor output signal is maintained. With the NTC sensor operating in the CCM mode there exists negative electrothermal feedback and question of instability in this particular case does not arise.

Note that the bolometer can be operated during a short interval of time at higher $L_{0}=1200 \mu \mathrm{A} / I_{\max }=1.53$, in presence of strong drift, without the risk of catastrophic damage. Explosive device destruction would occur at $L_{0}$ $=1530 \mu \mathrm{A} / I_{\max }>1.95$ and thus

$$
I_{\max }^{\text {critical }} \approx 1.95 \sqrt{G / \beta R_{0}} .
$$

\section{B. Constant voltage operation}

Figures 5(a)-5(f) display the simulated operating characteristics of the NTC sensor in the CVM mode, where $T_{s}$ is plotted as a function of time for fixed constant $G$ and $C$ (case 1). This is in response to a step change of $1 \mathrm{~mW}$ of incident radiation. Positive electrothermal feedback exists also in this case. In Figs. 5(g)-5(1) are shown the corresponding characteristics for temperature dependent $G\left(T_{s}\right)$ and $C\left(T_{S}\right)$, referred to as case 2 . Note that the $R\left(T_{s}\right)=A e^{B / T_{s}}$ was used for simulation studies. In either case, catastrophic thermal runaway occurs. For case 1, the explosive thermal runaway phenomenon is triggered for $V_{b}>5.8 \mathrm{~V}$ for case $1,37 \mathrm{~s}$ after the application of the radiation step. It happens approximately for $V_{b}>6.8 \mathrm{~V}$ in case 2 . The sensor temperature $T_{s}$ remains below $100 \mathrm{C}$ in either case. Slow runaway [see Fig. 5(e)] appears smoothly after about $80 \mathrm{~s}$ for case 1 and about $60 \mathrm{~s}$ [see Fig. 5(1)] for case 2, revealing nearly identical dynamic properties. The differences between cases 1 and 2 are marginal. With constant $G$ and $C$, the bias voltage causing runaway is calculated as $V_{\max }=8.66 \mathrm{~V}$ for $R_{0}=7 \mathrm{k} \Omega$. This value is approximately $3 \mathrm{~V}$ larger than the critical voltage obtained 

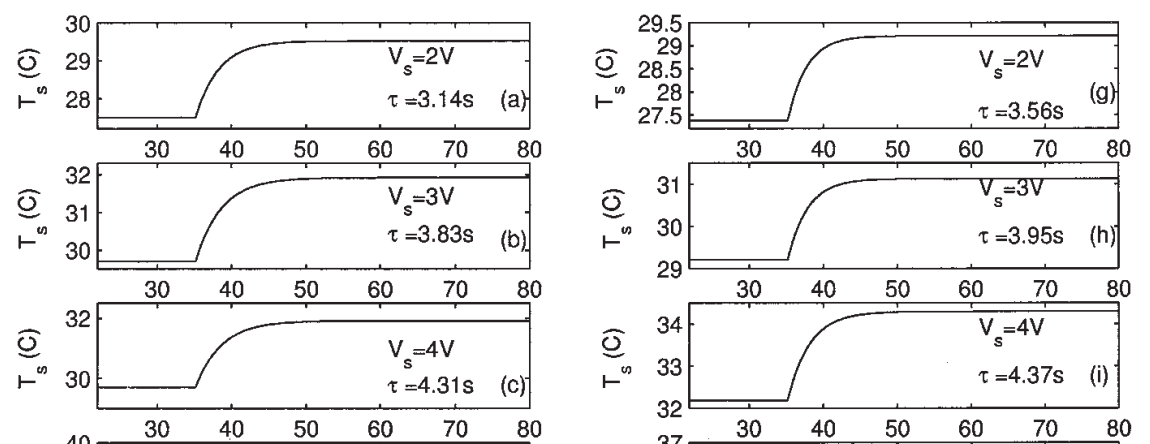

FIG. 5. Evolution of sensor temperature $T_{s}$ as a function of time for the NTC sensor for various bias voltages and $1 \mathrm{~mW}$ step incident radiation (simulation). Case 1, with constant $\mathrm{G}$ and $C$ values $[5(\mathrm{a})-5(\mathrm{f})]$. Case 2 for temperature dependent $G\left(T_{s}\right)$ and $C\left(T_{s}\right)[5(\mathrm{~g})-5(\mathrm{l})]$, as taken from Figs. 2(a)-2(d). The effective time constant for each bias voltage indicated in the figures.
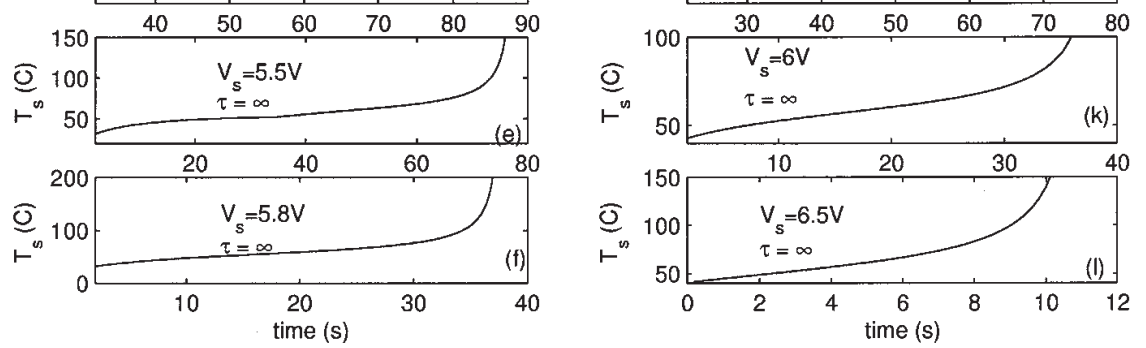

from the numerical solution of Eq. (1) as shown in Figs. 5(e)-5(f). This difference results from the more accurate numerical solution, which takes into account the exponential $R\left(T_{s}\right)$ characteristics of the NTC material rather than the linear approximation used in the analytical expression (3) for cases 1 and 2 .

\section{Sensor stability conditions}

The drift in the NTC sensor temperature also can be used as the limiting criterion for the maximum permitted bias voltage. The limiting bias voltage for stable operation can be extracted from Fig. 6. At $V_{b}=1 \mathrm{~V}$, the temperature drift is experimentally observed to be only approximately $0.1 \mathrm{mC} / \mathrm{s}$ as predicted by simulation. As before, a safety factor can be determined in terms of Eq. (11) as $L_{0}=1 \mathrm{~V} / V_{\max }=0.11$

$$
V_{\max }^{\text {stable }} \approx 0.11 \sqrt{-G R_{0} / \beta}
$$

for which a stable sensor output current is obtained. In terms of Eq. (11), without further safety measures, explosive device damage would occur at $L_{0}=6 \mathrm{~V} / V_{\max }=0.69$ and

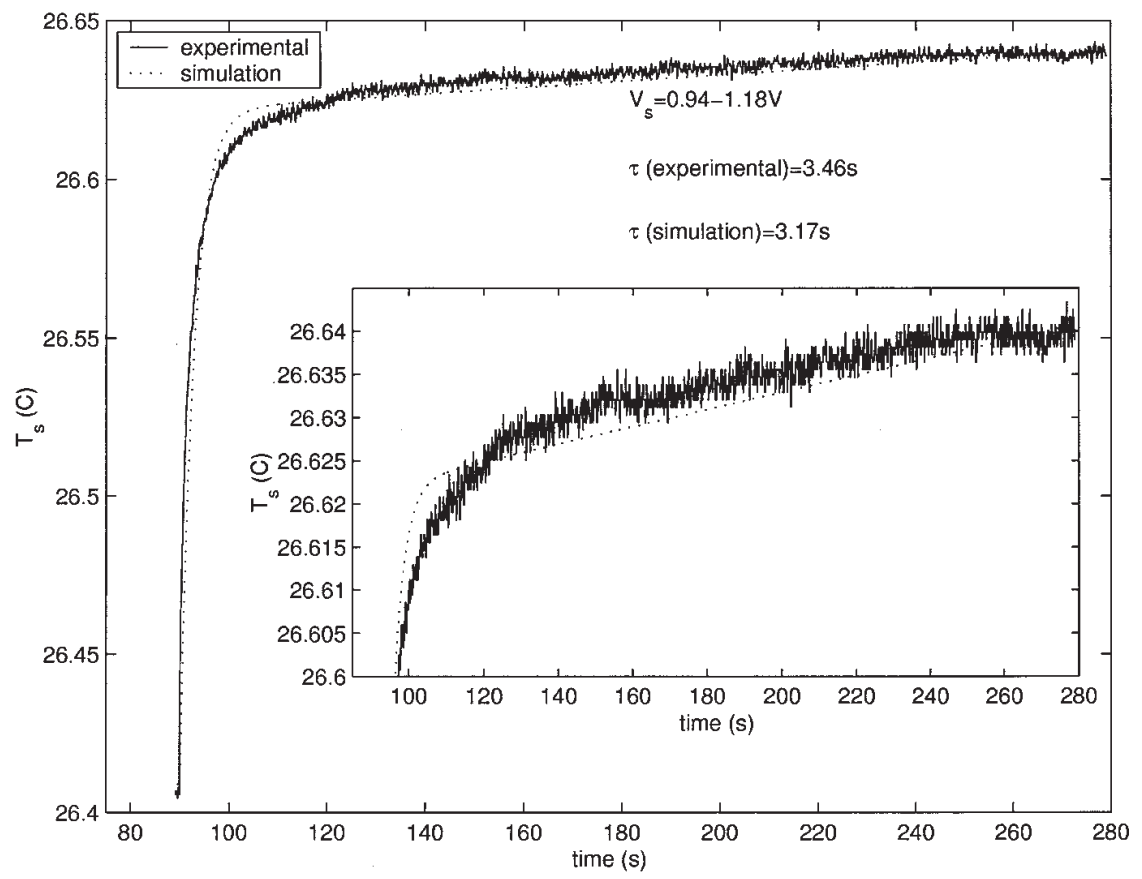

FIG. 6. Experimental (solid line) and calculated response curve (broken line) of the NTC sensor, for input step electrothermal excitation of $240 \mathrm{mV}$ on the top of $0.94 \mathrm{~V}$. Inset illustrates the appearance of an output signal drift of $0.062 \mathrm{mC} / \mathrm{s}$ for this condition. Note the signal fluctuation and a relatively noisy signal of the sensor. 


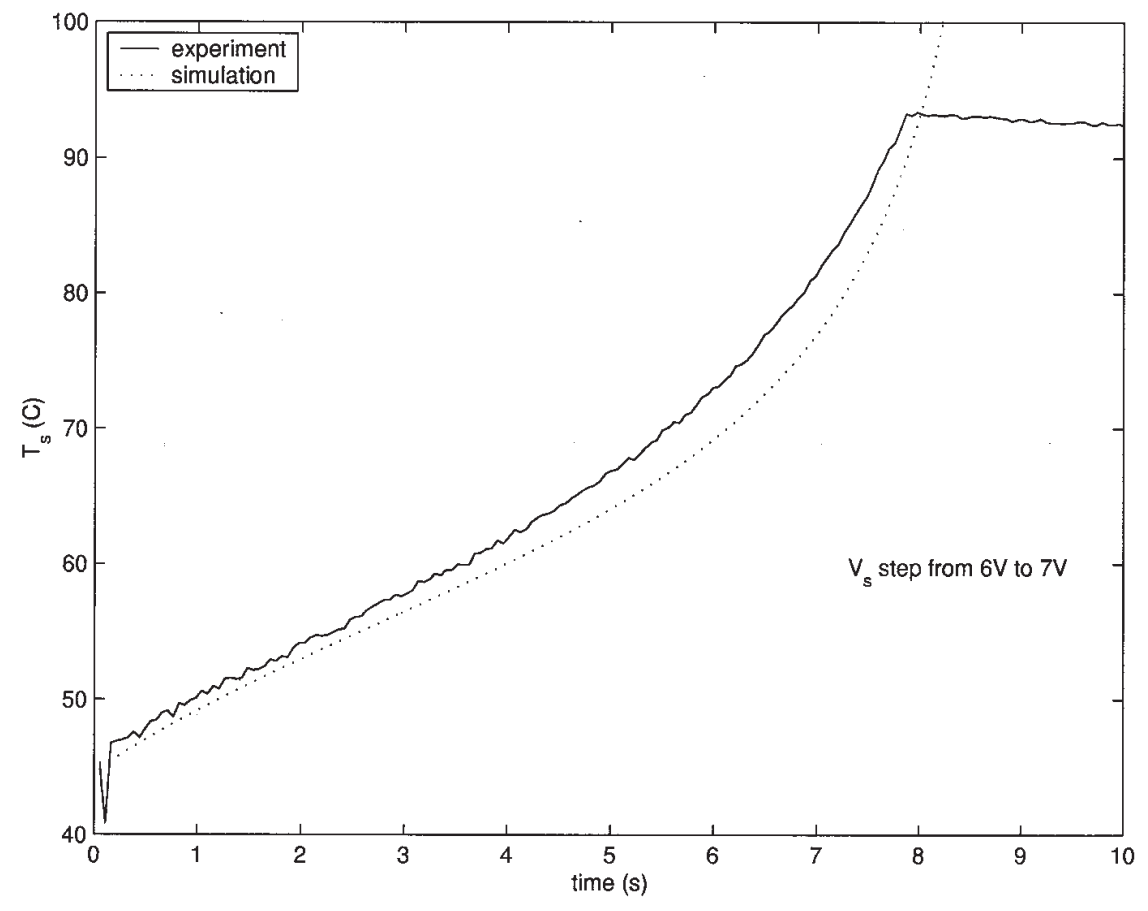

FIG. 7. Onset of thermal runaway for NTC sensor for a step of $1 \mathrm{~V}$ on the top of $6 \mathrm{~V}$. Calculated data are displayed in broken line. Output signal clamping is due to amplifier saturation.

$$
V_{\max }^{\text {critical }} \approx 0.69 \sqrt{-G R_{0} / \beta} .
$$

Unlike the situation for the PTC sensor, operating in the $\mathrm{CCM}$, in this case $T_{s}$ consistently remains much below the melting temperature of the semiconducting metal oxides, used as the NTC sensor material. Under the conditions of negative feedback, existing in the CVM operation with the PTC sensor, the operation is unconditionally stable and the time constant decreases with increasing bias voltage.

Figure 7 illustrates the experimentally obtained and simulated dynamic characteristics of the thermal runaway phenomenon for the NTC sensor. Radiation excitation was electrically simulated, by applying a voltage step of $1 \mathrm{~V}$, added to a constant bias voltage of $6 \mathrm{~V}$, representing approximately $0.15 \mathrm{~mW}$ of radiation power. For simulation studies we used the set of parameters corresponding to case 2 of Fig. 5(1). Experimental and simulated data agree within a tolerance of a few percent. This confirms the quality of the proposed model for describing the temperature dependence of $G$ and $C$ parameters. $T_{s}$ increases exponentially with time and the saturation of the amplifier output [see Fig. 1(b)] limits the sensor temperature reading to $95 \mathrm{C}$. The thermal runaway manifests approximately $10 \mathrm{~s}$ after the application of the electrically simulated radiation power. If the radiation power is reduced to $0.07 \mathrm{~mW}$ the delay for onset of thermal runaway is significantly increased to approximately $60 \mathrm{~s}$. It is evident from the data that the closer the bias voltage is to the theoretical maximum voltage, earlier the thermal runaway occurs.

Table II lists the values of critical biasing conditions and associated $L_{0}$ values for different operational behaviors. As shown by the present data, the safety factor $L_{0}$ for stable device operation needs to be chosen for both the PTC and NTC type thermoresistive sensors to be $\ll 0.5$. The safety margin is about a factor of 3 lower for the NTC type sensor.
Most likely, this is related to the difference in thermal capacities of the heat sinks of the two sensors under investigation. For microsensors with membrane type heat sensing elements, the thermal capacity $C_{\mathrm{HS}}$ of the effective heatsink, largely exceeds the heat capacity of the heat sensing membrane $C_{\mathrm{DET}}$, and the ratio $C_{\mathrm{HS}} / C_{\mathrm{DET}}$ is commonly $\geqslant 10^{4}$. This ratio is much smaller for the NTC sensor, where only the leads for electrical connection form the heat sink. Values of $L_{0}$ as low as 0.1 for stable operation were also observed experimentally for the widely used, and commercially available composite $\mathrm{Pt}_{100}$ sensor with PTC characteristics, which features a similar design as the NTC sensor under study. The study shows that the relations defining sensor instability can be used to define the operational limits, provided the appropriate safety margins as given by the safety factor $L_{0}$ are used.

There possibly exist other physical phenomena, associated with the positive electrothermal feedback. Examples are the occurrence of dielectric breakdown in high voltage insulators, voltage breakdown in the gate dielectrics of field effect transistors, the varistor operation, and electrothermal breakdown in gas discharges. Eventually, positive electrothermal feedback can be used to electrically trigger fast optical limiters, relying on the first order martensitic phase transition in metal oxides. ${ }^{5}$ Solid insulators typically display a weak NTC characteristics. However, besides the thermal

TABLE II. Critical limits for sensor operation.

\begin{tabular}{lcccc}
\hline \hline Sensor & Stable operation & Signal drift & $\begin{array}{c}\text { Explosive } \\
\text { destruction }\end{array}$ & Critical bias \\
\hline NTC & $L_{0}<0.11$ & $0.11<L_{0}<0.69$ & $L_{0}>0.69$ & $V_{b}=8.66 \mathrm{~V}$ \\
PTC & $L_{0}<0.35$ & $0.35<L_{0}<1.53$ & $L_{0}>1.95$ & $I_{b}=783 \mu \AA$ \\
\hline \hline
\end{tabular}


breakdown, the dielectric breakdown phenomenon involves several other mechanisms: hot carrier injection from the metal electrodes at very high electric fields by tunneling, carrier injection by detrapping of charge carriers from deep trap states in the volume of the samples, and current runaway by carrier multiplication through electron-hole pair generation through field acceleration of free carriers in the volume of the sample. ${ }^{16}$ The influence of thermal runaway on voltage breakdown in gate dielectrics is presently under investigation.

\section{ACKNOWLEDGMENTS}

Thanks are due to CNPq, CAPES, and DAAD for the award of research and study fellowship during the course of this study. The authors also thank Dr. Mang Ou-Yang from Precision Instrument Center, Hsinchu, Taiwan for providing us with the PTC sensor and Dr. Kenzo Watanabe from Shizuoka University, Hamamatsu, Japan for the donation of the NTC sensor.

${ }^{1}$ M. J. M. E. de Nivelle et al., J. Appl. Phys. 82, 4719 (1997).

${ }^{2}$ H. Neff, A. M. N. Lima, G. S. Deep, R. C. S. Freire, E. Melcher, I. A. Khrebtov, and A. D. Tkachenko, Appl. Phys. Lett. 76, 640 (2000).

${ }^{3}$ K. D. Irwin, Appl. Phys. Lett. 66, 1998 (1995).
${ }^{4}$ A. T. Lee, P. L. Richards, S. W. Nam, B. Cabrera, and K. D. Irwin, Phys. Lett. 69, 1801 (1996).

${ }^{5}$ L. A. L. de Almeida, G. S. Deep, A. M. N. Lima, and H. Neff, Appl. Phys. Lett. 77, 3654 (2000).

${ }^{6}$ Thermal Sensors, edited by G. C. M. Meijer and A. W. Herwaarden (Institute of Physics, Bristol, 1994).

${ }^{7}$ G. S. Deep, J. S. R. Neto, A. M. N. Neto, R. C. S. Freire, and P. C. Lobo, IEEE Trans. Instrum. Meas. 45, 332 (1996).

${ }^{8}$ H. Neff, M. Berg, and R. Barth, IEEE Trans. Appl. Supercond. 7, 3802 (1997).

${ }^{9}$ J. S. Shie and P. K. Weng., IEEE Int. Conf. Solid-State Sensors Actuators Transducer'91, 1991, p. 627.

${ }^{10}$ E. R. G. Eckert and R. M. Drake, Analysis of Heat and Mass Transfer (McGraw-Hill, New York, 1972).

${ }^{11}$ G. B. Brandão, G. S. Deep, A. M. N. Lima, and H. Neff, in Conf. Rec. of the XIII Congresso Brasileiro de Automática, Florianópolis, SC, 2000, p. 2372 .

${ }^{12}$ G. S. Deep, A. Oliveira, R. C. S. Freire, J. S. R. Neto, and M. B. M. França, in Conf. Rec. of the Intern. Conf. on Electronics, Circuits and Systems (ICECS), 1999, p. 1301.

${ }^{13}$ M. Ou-Yang, C. S. Sheen, and J. S. Shie, IEEE Trans. Instrum. Meas. 47, 403 (1998).

${ }^{14}$ I. A. Khrebtov, A. D. Tkachenko, E. Steinbeiss, and W. Michalke, in Conf. Rec. of 4th European Workshop on Low Temperature Electronics (WOLTE-4), ESA, WPP-171, 2000, p. 335.

${ }^{15}$ E. Monticone, L. Boarino, G. Lerondel, R. Steni, G. Amato, and V. Lacquaniti, Appl. Surf. Sci. 142, 267 (1999).

${ }^{16}$ H. Neff and P. Lange, J. Appl. Phys. 72, 4369 (1992). 